GANIT J. Bangladesh Math. Soc. (ISSN 1606-3694) 32 (2012) 23-28

\title{
ON DERIVATIONS IN PRIME GAMMA-NEAR-RINGS
}

\author{
Kalyan Kumar Dey ${ }^{1}$ and Akhil Chandra Paul ${ }^{2}$
}

Department of Mathematics

University of Rajshahi, Rajshahi-6205, Bangladesh

Email: ${ }^{1}$ kkdmath@yahoo.com and ${ }^{2}$ acpaulrubd_math@yahoo.com

Received 30.11.2011 Accepted 14.07.2012

\begin{abstract}
Let $N$ be a non zero-symmetric left $\Gamma$-near-ring. If $N$ is a prime $\Gamma$-near-ring with nonzero derivations $D_{1}$ and $D_{2}$ such that $D_{1}(x) \breve{U} D_{2}(y)=D_{2}(x) \breve{U} D_{1}(y)$ for every $x, y \in N$ and $\breve{U} \in \Gamma$, then we prove that $N$ is an abelian $\Gamma$-near-ring. Again if $N$ is a 2-torsion free prime $\Gamma$-near-ring and $D_{1}$ and $D_{2}$ are derivations satisfying $D_{1}(x) \breve{U} D_{2}(y)=$ $D_{2}(x) \breve{U} D_{1}(y)$ for every $x, y \in N$ and $\breve{U} \in \Gamma$, then we prove that $D_{1} D_{2}$ is a derivation on $N$ if and only if $D_{1}=0$ or $D_{2}=0$.
\end{abstract}

Key words: Prime $\Gamma$-near-rings, semiprime $\Gamma$-near-rings, $N$-subsets, derivations.

2010 Mathematics Subject Classification. 16Y30

\section{Introduction}

In [2] Bell and Mason introduced the notion of derivations in near-rings. They obtained some basic properties of derivations in near-rings. Then Mustafa [11] investigated some commutativity conditions for a $\Gamma$-near-ring with derivations. Cho [5] studied some characterizations of $\Gamma$-near-rings and some regularity conditions. In classical ring theory, Posner [9], Herstein [6], Bergen [4], Bell and Daif [1] studied derivations in prime and semiprime rings and obtained some commutativity properties of prime rings with derivations. In near ring theory, Bell and Mason [2], and also Cho [10] worked on derivations in prime and semiprime near-rings.

In this paper, we slightly extend the results of Cho [10] in prime $\Gamma$-near-rings with certain conditions by using derivations.

A $\Gamma$-near-ring is a triple $(N,+, \Gamma)$ where

(i) $(N,+)$ is a group (not necessarily abelian),

(ii) $\Gamma$ is a non-empty set of binary operations on $N$ such that for each $\breve{U} \in \Gamma,(N,+, \breve{U})$ is a left near-ring.

(iii) $a \breve{U}(b \overline{\mathrm{D}} c)=(a \breve{U} b) \overline{\mathrm{b}} c$, for all $a, b, c \in N$ and $\breve{\mathrm{U}} \mathrm{b} \in \Gamma$.

Exactly speaking, it is a left $\Gamma$-near-ring because it satisfies the left distributive law. We will use the word $\Gamma$-near-ring to mean left $\Gamma$-near-ring. For a near-ring $N$, the set $N_{0}=$ $\{a \in N$ : $0 \breve{U} a=0, \breve{U} \in \Gamma\}$ is called the zero-symmetric part of $N$. А $\Gamma$-near-ring $N$ is said to be zero-symmetric if $N=N_{0}$. Throughout this paper, $N$ will denote a zero-symmetric left 
$\Gamma$-near-ring. А $\Gamma$-near-ring $N$ is called a prime $\Gamma$-near-ring if $N$ has the property that for $a$, $b \in N, a \Gamma N \Gamma b=\{0\}$ implies $a=0$ or $b=0 . N$ is called a semiprime $\Gamma$-near-ring if $N$ has the property that for $a \in N, a \Gamma N \Gamma a=\{0\}$ implies $a=0$. A nonempty subset $U$ of $N$ is called a right $N$-subset (resp. left $N$-subset) if $U \Gamma N \subset U$ (resp. $N \Gamma U \subset U$ ), and if $U$ is both a right $N$-subset and a left $N$-subset, it is said to be an $N$-subset of $N$. An ideal of $N$ is a subset $I$ of $N$ such that (i) $(I,+)$ is a normal subgroup of $(N,+)$, (ii) $a \Gamma(I+b)-a \Gamma b \subset I$ for all $a, b \in N$, (iii) $(I+a) \Gamma b-a \Gamma b \subset I$ for all $a, b \in N$. If $I$ satisfies (i) and (ii) then it is called a left ideal of $N$. If $I$ satisfies (i) and (iii) then it is called a right ideal of $N$.

On the other hand, a (two-sided) $N$-subgroup of $N$ is a subset $H$ of $N$ such that

(i) $(H,+)$ is a subgroup of $(N,+)$, (ii) $N \Gamma H \subset H$, and (iii) $H \Gamma N \subset H$. If $\mathrm{H}$ satisfies (i) and (ii) then it is called a left $N$-subgroup of $N$. If $H$ satisfies (i) and (iii) then it is called a right $N$-subgroup of $N$. Note that normal $N$-subgroups of $N$ are not equivalent to ideals of $N$. Every right ideal of $N$, right $N$-subgroup of $N$ and right semigroup ideal of $N$ are right $N$-subsets of $N$, and symmetrically, we can apply for the left case. A derivation $D$ on $N$ is an additive endomorphism of $N$ with the property that for all $a, b \in N$ and $\breve{U} \in \Gamma, D(a \breve{U} b)=$ $a \breve{U} D(b)+D(a) \breve{U} b$.

\section{Derivations in prime $\Gamma$-near-rings}

A $\Gamma$-near-ring $N$ is called abelian if $(N,+)$ is abelian, and 2-torsion free if for all $a \in N, 2 a$ $=0$ implies $a=0$.

Lemma 2.1. Let $D$ be an arbitrary additive endomorphism of $N$. Then $D(a \breve{U} b)=a \breve{U} D(b)+$ $D(a) \breve{U} b$ if and only if $D(a \breve{U} b)=D(a) \breve{U} b+a \breve{U} D(b)$ for all $a, b \in N$ and $\breve{U} \in \Gamma$.

Proof. Suppose that $D(a \breve{U} b)=a \breve{U} D(b)+D(a) \breve{U} b$, for all $a, b \in N$ and $\breve{U} \in \Gamma$. For $\breve{U} \in \Gamma$ and from

$a \breve{U}(b+b)=a \breve{U} b+a \breve{U} b$ and $N$ satisfies left distributive law

$D(a \breve{U}(b+b))=a \breve{U} D(b+b)+D(a) \breve{U}(b+b)=a \breve{U}(D(b)+D(b))+D(a) \breve{U} b+D(a) \breve{U} b$

$=a \breve{U} D(b)+a \breve{U} D(b)+D(a) \breve{U} b+D(a) \breve{U} b$

and

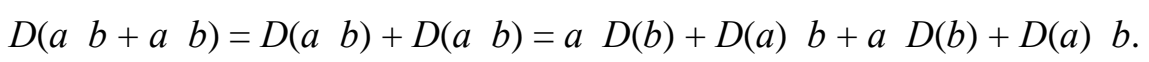

Comparing these two equalities, we have $a \breve{U} D(b)+D(a) \breve{U} b=D(a) \breve{U} b+a \breve{U} D(b)$. Hence $D(a \breve{U} b)=D(a) \breve{U} b+a \breve{U} D(b)$, for $a, b \in N, \breve{U} \in \Gamma$.

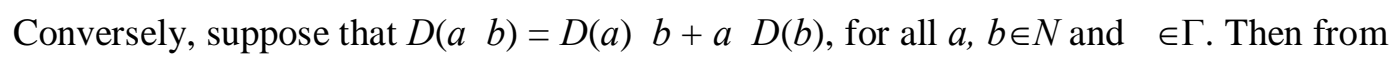
$D(a \breve{U}(b+b))=D(a \breve{U} b+a \breve{U} b)$ and the above calculation of this equality, we can induce that $D(a \breve{U} b)=a \breve{U} D(b)+D(a) \breve{U} b$, for $a, b \in N, \breve{U} \in \Gamma$.

Lemma 2.2. Let $D$ be a derivation on $N$. Then $N$ satisfies the following right distributive laws: for all $a, b, c \in N$ and $\breve{\mathrm{U}}, \mathrm{b} \in \Gamma$,

$$
\begin{aligned}
& \{a \breve{\mathrm{U} D}(b)+D(a) \breve{U} b\} \overline{\mathrm{b}} c=a \breve{\mathrm{U}} D(b) \overline{\mathrm{b}} c+D(a) \breve{U} b \overline{\mathrm{b}} c, \\
& \{D(a) \breve{U} b+a \breve{\mathrm{U}} D(b)\} \overline{\mathrm{b}} c=D(a) \breve{U} b \overline{\mathrm{b}} c+a \breve{U} D(b) \overline{\mathrm{b}} c,
\end{aligned}
$$


Proof. From the calculation for $D((a \breve{U} b) \overleftarrow{b} c)=D(a \breve{U}(b \bar{b} c))$ for all $a, b, c \in N$ and $\breve{\mathrm{U}} \overline{\mathrm{b}} \in \Gamma$ and Lemma 2.1, we can induce our result.

Lemma 2.3. Let $N$ be a prime $\Gamma$-near-ring and let $U$ be a nonzero $N$-subset of $N$. If $a$ be an element of $N$ such that $U \Gamma a=\{0\}$ (or $a \Gamma U=\{0\}$ ), then $a=0$.

Proof. Since $U \neq\{0\}$, there exist an element $u \in U$ such that $u \neq 0$. Consider that

$u \Gamma N \Gamma a \subset U \Gamma a=\{0\}$. Since $u \neq 0$ and $N$ is a prime $\Gamma$-near-ring, we have that $a=0$.

Corollary 2.4. Let $N$ be a semiprime $\Gamma$-near-ring and let $U$ be a nonzero $N$-subset of $N$. If $a$ be an element of $N(U)$ such that $U \Gamma a \Gamma a=\{0\}$ (or $a \Gamma a \Gamma U=\{0\}$ ), where $N(U)$ is the normalizer of $U$, then $a=0$.

Lemma 2.5. Let $N$ be a prime $\Gamma$-near-ring and $U$ a nonzero $N$-subset of $N$. If $D$ is a nonzero derivation on $N$. Then (i) If $a, b \in N$ and $a \Gamma U \Gamma b=\{0\}$, then $a=0$ or $b=0$.

(ii) If $a \in N$ and $D(U) \Gamma a=\{0\}$, then $a=0$. (iii) If $a \in N$ and $a \Gamma D(U)=\{0\}$, then $a=0$.

Proof. (i) Let $a, b \in N$ and $a \Gamma U \Gamma b=\{0\}$. Then $a \Gamma U \Gamma N \Gamma b \subset a \Gamma U \Gamma b=\{0\}$. Since $N$ is a prime $\Gamma$-near-ring, $a \Gamma U=0$ or $b=0$.

If $b=0$, then we are done. So if $b \neq 0$, then $a \Gamma U=0$. Applying Lemma 2.3, $a=0$.

(ii) Suppose $D(U) \Gamma a=\{0\}$, for $a \in N$. Then for all $u \in U$ and $b \in N$, from Lemma 2.2, we have for all $a, b \in N$ and $\breve{\mathrm{U}}, \mathrm{b} \in \Gamma, 0=D(b \breve{U} u) \overline{\mathrm{b}} a=(b \breve{\mathrm{U}} D(u)+D(b) \breve{U} u) \overline{\mathrm{b}} a=b \breve{\mathrm{U}} D(u) \overline{\mathrm{b}} a+$ $D(b) \breve{u} \measuredangle a=D(b) \breve{u} \iota \mathrm{\Xi} a$. Hence $D(b) \Gamma U \Gamma a=\{0\}$ for all $b \in N$. Since $D$ is a nonzero derivation on $N$, we have that $a=0$ by the statement (i).

(iii) Suppose $a \Gamma D(U)=\{0\}$ for $a \in N$. Then for all $u \in U, b \in N$ and $\breve{\mathrm{U}} \mathrm{\complement} \in \Gamma$,

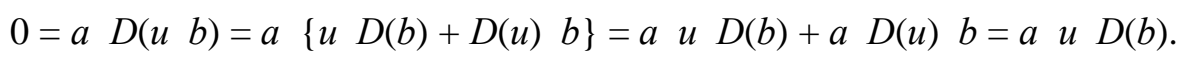

Hence $a \Gamma U \Gamma D(b)=\{0\}$ for all $b \in N$. From the statement (i) and $D$ is a nonzero derivation on $N$, we have that $a=0$.

We remark that to obtain any of the conclusions of Lemma 2.5, it is not sufficient to assume that $U$ is a right $N$-subset, even in the case that $N$ is a $\Gamma$-ring.

Theorem 2.7. Let $N$ be a prime $\Gamma$-near-ring and $U$ be a right $N$-subset of $N$. If $D$ is a nonzero derivation on $N$ such that $D^{2}(U)=0$, then $D^{2}=0$.

Proof. For all $u, v \in U$ and $\breve{U} \in \Gamma$, we have $D^{2}(u \breve{U} v)=0$. Then

$0=D^{2}(u \breve{U})=D(D(u \breve{U}))=D\{D(u) \breve{U} v+u \breve{U} D(v)\}$

$=D^{2}(u) \breve{U} v+D(u) \breve{U} D(v)+D(u) \breve{U} D(v)+u \breve{U} D^{2}(v)$

$=D^{2}(u) \breve{U} v+2 D(u) \breve{U} D(v)+u \breve{U} D^{2}(v)$.

Thus $2 D(u) \Gamma D(U)=\{0\}$ for all $u \in U$. From Lemma 2.5(iii), we have $2 D(u)=0$.

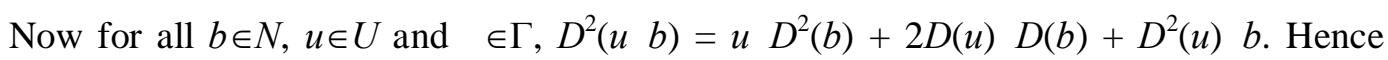
$U \Gamma D^{2}(b)=\{0\}$ for all $b \in N$. By Lemma 2.3, we have $D^{2}(b)=0$ for all $b \in N$. Consequently $D^{2}=0$.

Lemma 2.8. Let $D$ be a derivation of a prime $\Gamma$-near-ring $N$ and $a$ be an element of $N$. If $a \breve{\mathrm{U}}(x)=0$ (or $D(x) \breve{U} a=0$ ) for all $x \in N, \breve{\cup} \in \Gamma$, then either $a=0$ or $D$ is zero. 
Proof. Suppose that $a \breve{\mathrm{U}} D(x)=0$ for all $x \in N$, Ŭ $\in \Gamma$. Replacing $x$ by $x \mathrm{~b} y$, (for all $\mathrm{b} \in \Gamma$ ) we have that $a \breve{\mathrm{U}} D(x \overline{\mathrm{b}} y)=0=a \breve{\mathrm{U}} D(x) \overline{\mathrm{b}} y+a \breve{\mathrm{U}} x \mathrm{\sigma} D(y)$ by Lemma 2.2. Then $a \mathrm{U} x \mathrm{~b} D(y)=0$ for all $x, y \in N, \breve{\mathrm{U}} \mathrm{\textrm {b }} \in \Gamma$.

If $D$ is not zero, that is, if $D(y) \neq 0$ for some $y \in N$, then, since $N$ is a prime $\Gamma$-near-ring, $a \Gamma N \Gamma D(y)$ implies that $a=0$.

Now we prove our main result.

Theorem 2.9. Let $N$ be a $\Gamma$-prime near-ring with nonzero derivations $D_{1}$ and $D_{2}$ such that for all $x, y \in N$ and $\breve{U} \in \Gamma, D_{1}(x) \breve{U} D_{2}(y)=-D_{2}(x) \breve{U} D_{1}(y)$

Then $N$ is an abelian $\Gamma$-near-ring.

Proof. Let $x, u, v \in N, \breve{U} \in \Gamma$. From the condition (1), we obtain that

$0=D_{1}(x) \breve{U} D_{2}(u+v)+D_{2}(x) \breve{U} D_{1}(u+v)$

$\left.\left.=D_{1}(x) \breve{\mathrm{U}} D_{2}(u)+D_{2}(v)\right]+D_{2}(x) \breve{U} D_{1}(u)+D_{1}(v)\right]$

$=D_{1}(x) \breve{U} D_{2}(u)+D_{1}(x) \breve{U} D_{2}(v)+D_{2}(x) \breve{U} D_{1}(u)+D_{2}(x) \breve{U} D_{1}(v)$

$=D_{1}(x) \breve{U} D_{2}(u)+D_{1}(x) \breve{U} D_{2}(v)-D_{1}(x) \breve{U} D_{2}(u)-D_{1}(x) \breve{U} D_{2}(v)$

$=D_{1}(x) \breve{U}\left[D_{2}(u)+D_{2}(v)-D_{2}(u)-D_{2}(v)\right]=D_{1}(x) \breve{U} D_{2}(u+v-u-v)$.

Thus $D_{1}(N) \Gamma D_{2}(u+v-u-v)=\{0\}$.

By Lemma 2.8, we have $D_{2}(u+v-u-v)=0$.

Now, we substitute $x \mathrm{D} u$ and $x \mathrm{~b} v(\mathrm{~b} \in \Gamma)$ instead of $u$ and $v$ respectively in (3). Then from Lemma 2.1, we deduce that for all $x, u, v \in N, \mathrm{~b} \in \Gamma$,

$0=D_{2}(x \mathrm{\sigma} u+x \mathrm{\sigma} v-x \mathrm{\sigma} u-x \mathrm{\sigma} v)=D_{2}[x \mathrm{\sigma}(u+v-u-v)]$

$=D_{2}(x) \mathfrak{\mathrm { D }}(u+v-u-v)+x \mathrm{E} D_{2}(u+v-u-v)=D_{2}(x) \mathrm{b}(u+v-u-v)$.

Again, applying Lemma 2.8, we see that for all $u, v \in N, u+v-u-v=0$.

Consequently, $N$ is an abelian $\Gamma$-near-ring.

Theorem 2.10. Let $N$ be a prime $\Gamma$-near-ring of 2-torsion free and let $D_{1}$ and $D_{2}$ be derivations with the condition $D_{1}(a) \breve{\cup} D_{2}(b)=D_{2}(b) \breve{U} D_{1}(a)$

for all $a, b \in N$ and $\breve{U} \in \Gamma$ on $N$. Then $D_{1} D_{2}$ is a derivation on $N$ if and only if either $D_{1}=0$ or $D_{2}=0$.

Proof. Suppose that $D_{1} D_{2}$ is a derivation. Then we obtain for $\breve{\mathrm{U}} \in \Gamma$,

$D_{1} D_{2}(a \breve{U} b)=a \breve{U} D_{1} D_{2}(b)+D_{1} D_{2}(a) \breve{U} b$.

Also, since $D_{1}$ and $D_{2}$ are derivations, we get

$D_{1} D_{2}(a \breve{U} b)=D_{1}\left(D_{2}(a \breve{U} b)\right)=D_{1}\left(a \breve{U} D_{2}(b)+D_{2}(a) \breve{U} b\right)=D_{1}\left(a \breve{U} D_{2}(b)\right)+D_{1}\left(D_{2}(a) \breve{U} b\right)$

$=a \breve{U} D_{1} D_{2}(b)+D_{1}(a) \breve{U} D_{2}(b)+D_{2}(a) \breve{U} D_{1}(b)+D_{1} D_{2}(a) \breve{U} b$.

From (5) and (6) for $D_{1} D_{2}(a \breve{b} b)$ for all $a, b \in N$, Ч̌ $\in \Gamma, D_{1}(a) \breve{U} D_{2}(b)+D_{2}(a) \breve{U} D_{1}(b)=0$.

Hence from Theorem 2.9, we know that $N$ is an abelian $\Gamma$-near-ring. 
Replacing $a$ by $a \breve{U} \underline{D}_{2}(c)$ in (7), and using Lemma 2.1 and Lemma 2.2, we obtain that

$0=D_{1}\left(a \breve{U} D_{2}(\mathrm{c})\right) \breve{U} D_{2}(b)+D_{2}\left(a \breve{U} D_{2}(c)\right) \breve{U} D_{1}(b)$

$=\left\{D_{1}(a) \breve{U} D_{2}(c)+a \breve{U} D_{1} D_{2}(c)\right\} \breve{U} D_{2}(b)+\left\{a \breve{U} D_{2}^{2}(c)+D_{2}(a) \breve{U} D_{2}(c)\right\} \breve{U} D_{1}(b)$

$=D_{1}(a) \breve{U} D_{2}(c) \breve{U} D_{2}(b)+a \breve{U} D_{1} D_{2}(c) \breve{U} D_{2}(b)+a \breve{U} D_{2}^{2}(c) \breve{U} D_{1}(b)+D_{2}(a) \breve{U} D_{2}(c) \breve{U} D_{1}(b)$

$=D_{1}(a) \breve{U} D_{2}(c) 6 D_{2}(b)+a \breve{U}\left\{D_{1} D_{2}(c) \breve{U} D_{2}(b)+D_{2}^{2}(c) \breve{U} D_{1}(b)\right\}+D_{2}(a) \breve{U} D_{2}(c) \breve{U} D_{1}(b)$ :

On the other hand, replacing $a$ by $D_{2}(c)$ in (7), we see that

$D_{1}\left(D_{2}(c)\right) \breve{U} D_{2}(b)+D_{2}\left(D_{2}(c)\right) \breve{U} D_{1}(b)=0$.

This equation implies that $a \breve{U}\left\{D_{1} D_{2}(c) \breve{U} D_{2}(b)+D_{2}^{2}(c) \breve{U} D_{1}(b)\right\}=0$.

Hence, from the above last long equality, we have the following equality

$D_{1}(a) \breve{U} D_{2}(c) \breve{U} D_{2}(b)+D_{2}(a) \breve{U} D_{2}(c) \breve{U} D_{1}(b)=0$, for all $a, b, c \in N, \breve{U} \in \Gamma$.

Replacing $a$ and $b$ by $c$ in (7) respectively, we see that

$D_{2}(c) \breve{U} D_{1}(b)=-D_{1}(c) \breve{U} D_{2}(b), D_{1}(a) \breve{U} D_{2}(c)=-D_{2}(a) \breve{U} D_{1}(c)$.

So that (8) becomes

$0=\left\{-D_{2}(a) \breve{U} D_{1}(c)\right\} \breve{U} D_{2}(b)+D_{2}(a) \breve{U}\left\{-D_{1}(c) \breve{U} D_{2}(b)\right\}$

$\left.=D_{2}(a) \breve{U}\left(-D_{1}(c)\right) \breve{U} D_{2}(b)+D_{2}(a) \breve{U}-D_{1}(c)\right) \breve{U} D_{2}(b)$

$=D_{2}(a) \mathfrak{\mathrm { V }}\left\{\left(-D_{1}(c)\right) \breve{U} D_{2}(b)-D_{1}(c) \breve{U} D_{2}(b)\right\}$ for all $a, b, c \in N, \breve{U} \in \Gamma$. If $D_{2} \neq 0$, then by Lemma 2.8, we have the equality: $\left(-D_{1}(c)\right) \breve{U} D_{2}(b)-D_{1}(c) \breve{U} D_{2}(b)=0$,

that is, $D_{1}(c) \breve{U} D_{2}(b)=\left(-D_{1}(c)\right) \breve{U} D_{2}(b)$, for all $b, c \in N$, Ǔ $\in \Gamma$.

Thus, using the given condition of our theorem, we get

$\left.\left(-D_{1}(c)\right) \breve{U} D_{2}(b)=D_{1}(-c) \breve{U} D_{2}(b)=D_{2}(b) \breve{U} D_{1}(-c)=D_{2}(b) \breve{U}-D_{1}(c)\right)$

$=-D_{2}(b) \breve{U} D_{1}(c)=-D_{1}(c) \breve{U} D_{2}(b)$.

From (9) and (10) we have that, for all $b, c \in N, \breve{U} \in \Gamma, 2 D_{1}(c) \breve{U} D_{2}(b)=0$.

Since $N$ is of 2-torsion free, $D_{1}(c) \breve{U} D_{2}(b)=0$. Also, since $D_{2}$ is not zero, by Lemma 2.8 , we see that $D_{1}(c)=0$ for all $c \in N$. Therefore $D_{1}=0$. Consequently, either $D_{1}=0$ or $D_{2}=$ 0 .

The converse verification is obvious. Thus our proof is complete.

As a consequence of Theorem 2.10, we get the following important statement.

Corollary 2.11. Let $N$ be a prime $\Gamma$-near-ring of 2-torsion free, and let $D$ be a derivation on $N$ such that $D^{2}=0$. Then $D=0$.

\section{References}

[1] Bell H. E. and Daif M. N., On derivations and commutativity in prime rings, Acta. Math. Hungar. 66(4) (1995), 337-343.

[2] Bell H. E. and Mason G., On derivations in near-rings. In: Gerhard Betsch (Ed.), NearRings and Near-Fields, Proceedings of the conference held at the University of Tubingen, Tubingen, August 4-10, 1985 (pp. 31-35). Noth-Holland, Amsterdam, 1987. 
[3] Bell H. E. and Mason G., On derivations in near-rings and rings. Math. J. Okayama Univ. 34 (1992), 135-144.

[4] Bergen J., Derivations in prime rings. Canad. Math. Bull. 26 (1983), no. 3, 267-227.

[5] Cho, Y. U., A study on derivations in near-rings. Pusan Kyongnam Math. J., 12(1) (1996), 63-69.

[6] Herstein I. N., A note on derivations. Canad. Math. Bull. 21(3) (1978), 369-370.

[7] Meldrum J. D. P., Near-Rings and Their Links with Groups, Research Notes in Mathematics, 134. Pitman (Advanced Publishing Program), Boston-London-Melbourne, 1985. MR 88a:16068

[8] Pilz G., Near-rings, North-Holland Mathematics Studies, 23. North-Holland, Amsterdam, 1983.

[9] Posner, E. C., Derivations in prime rings. Proc. Amer. Math. Soc. 8 (1957), 1093-1100.

[10] Yong Uk Cho, Some conditions on derivations in prime near rings, J Korea Soc Math Educ Ser B Pure Appl Math 8(2) 2001, 145-152.

[11] Mustafa Asci, $\Gamma-(\sigma, \tau)$-Derivation on Gamma Near Ring, International Math Forum, 2(3), 2007, 97-102. 